\title{
Correcting Flaws in the Assignment of Nitrogen Chemical Environments in N-Doped Graphene
}

\author{
Marc Figueras, ${ }^{1}$ Ignacio J. Villar-Garcia, ${ }^{2}$ Francesc Viñes, ${ }^{1}$ Carmen Sousa, ${ }^{1}$ Victor A. \\ de la Peña $\mathrm{O}^{\prime}$ Shea ${ }^{2}$ and Francesc Illas ${ }^{1} *$ \\ 1) Departament de Ciència de Materials i Química Física \& Institut de Química \\ Teòrica i Computacional (IQTCUB), Universitat de Barcelona, c/Martí i Franquès 1, \\ 08028 Barcelona, Spain \\ 2) Photoactivated Processes Unit, IMDEA Energy Institute, Parque Tecnológico de \\ Móstoles, Avda. Ramón de la Sagra 3, 28935 Móstoles, Madrid, Spain.
}

\begin{abstract}
X-ray Photoelectron Spectroscopy (XPS) applied to N-doped graphene leads to a rather broad N(1s) core level signal that, based on different sources available in the literature, is most often interpreted by fitting the experimental spectra to three peaks. The resulting $\mathrm{N}(1 \mathrm{~s})$ features are assigned to graphitic, pyrrolic, and pyridinic nitrogen, even if these are far from being uniquely defined in the literature. This broadly accepted interpretation has been questioned by recent accurate Hartree-Fock calculations concluding that graphitic and pyrrolic $\mathrm{N}(1 \mathrm{~s})$ core level binding energies are too close to be distinguished. Consideration of models with $\mathrm{N}$ in other so far unexplored environments such as $\mathrm{N}$ dimers or $\mathrm{N}$ at defects show some variations in the calculated core level binding energies. However, these are not large enough to justify a third peak and suggest that the usual three peaks interpretation of the N(1s) XPS in N-doped graphene may be an artefact caused by the fitting procedure. New measurements have been carried out for samples of N-doped graphene and the obtained $\mathrm{N}(1 \mathrm{~s})$ spectra fitted to two or three peaks. It turns out that the spectra can be equally fitted using two or three peaks but only the former is consistent with the results of the unbiased ab initio calculations which calls for a revision of the usual assignment.
\end{abstract}

Keywords: N-doping, Graphene, X-ray photoelectron spectroscopy, core level binding energy, graphitic, pyridinic, pyrrolic.

*corresponding author: francesc.illas@ub.edu 


\section{Introduction}

Since the landmark work of Novoselov et al. ${ }^{1}$ isolating graphene, this material has triggered a revolution in materials science and opened the new world of two dimensional (2D) materials, with graphene being indeed its main protagonist. Although the initial work was firstly conceived as an academic curiosity, the recent years have witnessed a blooming of possible applications, ${ }^{2}$ bringing graphene to a different level of research. These technological applications emerge from its surprising and appealing physical properties, such as quantum electronic transport, high elasticity, extremely high electron/hole mobilities, and electrochemical modulation, among others, ${ }^{3}$ making it very promising towards electronic transport and applied coating materials, with deformation possibilities which may trigger changes in its electronic conductivity.

Taking advantage of the unprecedented quantum electronic transport exhibited by graphene, ${ }^{2}$ one of the most promising fields of application involves electronic devices posing this new material as a perfect substitute to silicon in microchips, improving in size and efficiency terms. However, graphene utilization in electronics presents some obstacles since its electronic structure is that of a semimetal featuring the well-known Dirac points in the reciprocal space, where energy gaps between valence and conduction bands vanish, rendering a metallic character yet with nominally zero charge carriers, ${ }^{4}$ even though close to these Dirac points, electrons and holes behave as massless charge carriers with ballistic transport. However, in order to be used as a transistor graphene needs to be modified in order to exhibit the characteristic small band gap of semiconductors. Consequently, large efforts have been dedicated to open a band gap in graphene without disrupting much of its electronic structure. In particular, doping has been frequently tried since this provides an extremely efficient way to tune material properties, including the electronic structure. Wang et $a l^{5}$ reported that thermally annealed graphene in ammonia resulted in substitution of carbon atoms by nitrogen thus leading to $\mathrm{N}$-doped graphene, a material exhibiting a promising field-effect transistor at room temperature. The existence of carbon-nitrogen bonds and the nature of the Ncontaining species was confirmed based on X-ray Photoelectron Spectroscopy (XPS) data. The authors suggested that the $\mathrm{N}$ atoms occupy mainly graphene edges, although the same authors recognized that the samples could be contaminated with oxygen. Other procedures have been proposed to obtain free $\mathrm{N}$-doped graphene in a more controlled environment. For instance, using arc discharge between carbon electrodes. ${ }^{6}$ 
Even though N-doped graphene displaying properties useful for transistors has been successfully synthesized, a better description of its composition is required to better control and modify the gap at will. XPS has been broadly used because shifts in the core level binding energy of a given chemical element provides direct information about its chemical environment such as oxidation states, ${ }^{7}$ even if a precise assignment requires additional information supplied either from experiment or theory. ${ }^{8,9}$ The XPS spectra of N-doped graphene depend somehow on the synthetic conditions and often consists of a rather broad signal as shown later in this work. Based on earlier experimental studies ${ }^{10}$ and on theoretical work for $\mathrm{N}$-containing carbonaceous materials published long before the discovery of graphene, ${ }^{11}$ it is customary to deconvolute the $\mathrm{N}(1 \mathrm{~s})$ XPS spectra of N-doped graphene into three contributions usually referred as graphitic, pyridinic, and pyrrolic nitrogen. ${ }^{12-19}$ However, the main conclusion of the paper by Casanovas et al. ${ }^{11}$ was that large $\mathrm{N}(1 \mathrm{~s})$ core level binding energies (BE) do not necessarily imply $\mathrm{N}$ bonded to $\mathrm{O}$ atoms, i.e., the presence of $\mathrm{N}$-oxide groups, rather than providing support to the assignment of XPS features to $\mathrm{N}$ at different sites in the graphitic network. Also, the calculated BEs were based on Hartree-Fock calculations where the so-called final state effects were indirectly taken into account by referring the calculated $\mathrm{N}(1 \mathrm{~s}) \mathrm{BEs}$ to the $\mathrm{C}(1 \mathrm{~s}) \mathrm{BE}$ arbitrarily set at $285 \mathrm{eV}$ and, hence, the reported values need to be taken with care. To make things worse, the meaning of graphitic, pyridinic, and pyrrolic nitrogen in the original work of Casanovas et al. ${ }^{11}$ and in the literature may differ significantly as discussed in detail in the next section.

Computational methods on proper models offer a suited framework to study these situations. While experimental procedures must deal with composition issues, theoretical models allow overcoming structural and composition problems defining totally controlled structures. Moreover, theory provides accurate estimate of the core level BEs and, in addition, allows determining the influence of initial and final state effects separately. ${ }^{8,20}$ A recent survey investigating a large number of core level BEs of molecules containing main group elements reported that total energy differences for the ground and the core ionized molecule predicted by the Hartree-Fock (HF) method —using the the so-called $\triangle \mathrm{SCF}$ approach-yield BEs and BEs shifts ( $\triangle \mathrm{BEs})$ with accuracies of 0.49 and $0.13 \mathrm{eV}$, respectively. ${ }^{21}$ Particularly for $\triangle \mathrm{BEs}$, the extendedly used Janak-Slater transition state approach yields accuracies just ranging $0.2-0.4 \mathrm{eV} .^{22}$ A recent theoretical study ${ }^{23}$ within the $\triangle \mathrm{SCF}$ approach has examined several graphene 
realistic models containing $\mathrm{N}$ and concluded that graphitic and pyrrolic $\mathrm{N}$ cannot be distinguished in the XPS spectra because their binding energies (BEs) are too similar. The consequences of this finding are clear, either a different type of $\mathrm{N}$ in graphene must be considered to explain the results emerging from fitting the experimental N(1s) XPS using three peaks, or, on the contrary, a fit with two peaks will be more appropriate. Indeed, a fit using two peaks has been used by some authors for other N-doped carbon materials ${ }^{24}$ and by a few studies on N-doped graphene. ${ }^{25,26}$

In the present work we solve the dilemma by a combination of theoretical models investigating alternative types of $\mathrm{N}$ in $\mathrm{N}$-doped graphene and experiments where the recorded XPS N(1s) spectra is fitted using two or three peaks. None of the investigated new possibilities is predicted to have N(1s) BEs justifying a third feature in the spectra. This is confirmed by the analysis of new XPS spectra recorded on different samples. We will present compelling evidence that the interpretation of the XPS N(1s) spectra in terms of graphitic, pyridinic and pyrrolic nitrogen is both biased and forced, and the consequences of the new interpretation are discussed.

\section{N-Doped graphene models, theoretical framework and computational details}

\section{Modelling N-Doped graphene}

Coronene and circumcoronene (Figure 1) are chosen as suitable models for graphene flakes where appropriate substitutions (see below) can lead to graphitic, pyridinic, and pyrrolic nitrogen as shown in Figure 2. The available literature defines graphitic nitrogen as the substitution of an inner $\mathrm{C}$ in the graphene layer by $\mathrm{N}^{12,13,15-17,19,27-31}$ In this way, the $\mathrm{N}$ atom is coordinated to three $\mathrm{C}$ atoms and contributes with two electrons to the $\pi$ system. There is also broad consensus in defining pyridinic $\mathrm{N}$ as the substitution of a $\mathrm{C}-\mathrm{H}$ in the graphene flake edge by a $\mathrm{N}$ atom. ${ }^{12-17,19,28,32-35}$ Thus pyridinic $\mathrm{N}$ is coordinated to two $\mathrm{C}$ atoms with the lone pair in the $\sigma$ framework and thus contributing to the $\pi$ system by one electron. The case of pyrrolic $\mathrm{N}$ requires some remarks since different authors use a different definition based on structural (Type 1), electron (Type 2), or both (Type 3) distribution criteria..$^{12-19,24-28,33,36}$ Type 1 pyrrolic $\mathrm{N}$ is defined as the substitution of a C-H in a five-membered ring by either $\mathrm{N}$ or $\mathrm{N}-\mathrm{H}$; in both cases $\mathrm{N}$ is coordinated to two $\mathrm{C}$ atoms and contributes to the $\pi$ system with one or two electrons, respectively. Type 2 pyrrolic $\mathrm{N}$ corresponds to the substitution of a $\mathrm{C}$ in the graphene flake edge by a $\mathrm{N}$ atom in a six or five-membered ring, thus Type 2 pyrrolic $\mathrm{N}$ is also 
coordinated to two $\mathrm{C}$ atoms and one $\mathrm{H}$ atom. Note that, regardless of the definition used for pyrrolic $\mathrm{N}$, this can only be encountered at edges or defects. The introduction of five-membered rings in the coronene and circumcoronene results in highly unstable structures. Therefore, additional models able to accommodate the strain introduced by the five-membered ring have been designed (Figure S1). It is worth pointing out that some authors define as pyridinic a $\mathrm{N}$ atom in a six-membered ring at the edge of the graphene flake bonded to two $\mathrm{C}$ atoms and one $\mathrm{H}$ atom. ${ }^{36}$ In the present work this situation corresponds to a Type 2 pyrrolic $\mathrm{N}$.

To search for alternative possibilities that can justify fitting the N(1) XPS spectra of $\mathrm{N}$-doped graphene to three well defined features, we considered the case of $\mathrm{N}$ dimers in the graphene flake. All possible combinations of two nearest-neighbour $\mathrm{N}$ atoms (nn) plus next nearest neighbour (nnn) have been considered. The configurations of $\mathrm{N}$ dimers can only be defined as graphitic, pyridinic, or pyrrolic when the two $\mathrm{N}$ atoms are of the same type (see examples in Figure 3). In any case, we will use the term graphitic, pyridinic or pyrrolic to denote the atom where a $\mathrm{N}(1 \mathrm{~s})$ core electron is ionized.

Finally, $\mathrm{N}$ at graphene native defects ${ }^{15,18,37-40}$ has also been considered. One of the most described defects is a double $\mathrm{C}$ vacancy. Here we consider adding a $\mathrm{N}$ atom thus forming a five-membered ring, while 2 edge $\mathrm{C}$ atoms are substituted by $2 \mathrm{~N}$ atoms (Figure 4A). Note that although containing 3 close $\mathrm{N}$ atoms, this system cannot be considered as a trimer because these $\mathrm{N}$ atoms are too far to show a significant and measurable interaction. Other types of $\mathrm{N}$ defects involve a double $\mathrm{C}$ vacancy, but with only one nitrogen added, bonded to a $\mathrm{H}$ atom (Figure 4B) or with $\mathrm{C}$ atoms bonded to $\mathrm{H}$ atoms (5C) although this last possibility leads to important surface torsion due steric effects. To complete the study we considered the so-called Stone-Wales ${ }^{41}$ defect involving neighbouring rings with 5 and 7 atoms (Figure 5A), substitution of a $\mathrm{C}$ atom by $\mathrm{N}$ in this defect leads to a new type of graphitic $\mathrm{N}$ (Figure $5 \mathrm{~B}$ ).

\section{Theoretical framework and computational details}

For each of the $\mathrm{N}$ doping situations in coronene and circumcoronene described in the previous subsection, the molecular structure has been obtained from geometry optimizations carried out by means of the B3LYP density functional theory based methods, whereas the N(1s) BEs have been obtained using the Hartree-Fock (HF) method. Both, B3LYP and HF calculations have been carried out using sufficiently 
large standard GTO basis sets. In particular, the $6-31 \mathrm{G}^{*}$ basis set $^{42}$ has been used for C and $\mathrm{H}$ whereas, to obtain accurate $\mathrm{N}(1 \mathrm{~s})$ binding energies, the more extended Alhrichs pVTZ basis set ${ }^{43,44}$ has been used to describe $\mathrm{N}$ as in previous work. ${ }^{23}$ The B3LYP method is known to provide structural parameters in very good agreement with experiment for a fairly large number of molecules including main group elements and transition metal complexes, ${ }^{45}$ whereas the HF method provides accurate enough BEs. ${ }^{21}$

The N(1s) binding energies have been calculated by HF total energy differences between the ground state of the corresponding $\mathrm{N}$-doped graphene model and the energy of the same system at the same geometry but with one electron ionized from the $\mathrm{N}(1 \mathrm{~s})$ core at the Hartree-Fock level; i.e. the $\Delta$ SCF approach. ${ }^{8,20,46}$ For interpretation purposes we also considered the estimates provided by Koopman's theorem. The later are usually referred to as initial state BEs, since they correspond to the ionization of the core electrons neglecting the response of the electron density to the core hole. Initial state effects reflect the environment of the atom to be core ionized before ionization. Final state effects account for the relaxation of the electron density in response to the presence of the core hole and are accounted for by the $\triangle \mathrm{SCF}$ approach. Usually $\triangle \mathrm{SCF}$ provide values in fairly good agreement with experiment since missing electron correlation and relativistic effects are of the order of a few tens of $\mathrm{eV}$ and tend to cancel each other. ${ }^{46}$

The B3LYP geometry optimization and $\triangle \mathrm{SCF}$ calculations have been carried out using the GAMESS program. ${ }^{47}$ Special care is needed to converge to the state of the cation with a core hole, as highlighted by Bagus more than 50 years ago. ${ }^{48}$ In the present work we used an alternative strategy leading to the same results as shown in previous work. $^{23}$

\section{N-Doped graphene synthesis and XPS measurements}

\section{Sample synthesis}

$\mathrm{N}$-doped graphene was synthesised following a procedure similar to Pendashteh et al. ${ }^{49}$ For the preparation, $32 \mathrm{mg}$ of Aldrich Graphene Oxide (GO) powder were re-dispersed in $30 \mathrm{~mL}$ of absolute ethanol using an ultrasound probe during $15 \mathrm{~min}$ (HielscherUP400S ultrasonic processor, Germany) until a stable dispersion was obtained. After that, $2 \mathrm{~mL}$ of a $\mathrm{NH}_{4} \mathrm{OH}$ solution $(28.0-30.0 \%$ ) was added to the $\mathrm{GO}$ dispersion under vigorous stirring. The reaction was kept under stirring at $80{ }^{\circ} \mathrm{C}$ overnight. The mixture was then transferred to a Teflon-lined hydrothermal autoclave 
reactor $\left(50 \mathrm{~mL}\right.$ of total capacity) and the reaction was continued at $150{ }^{\circ} \mathrm{C}$ for $3 \mathrm{~h}$. The resulting precipitate was collected by centrifugation at 6000 r.p.m. for 15 min after washing with pure ethanol and Mili-Q water. Finally, the sample was dried at $70{ }^{\circ} \mathrm{C}$ overnight. The $\mathrm{N}$ content was determined to $10.1 \pm 1.5 \%$ weight by elemental analysis.

\section{XPS Data Collection and sample mounting}

XPS spectra were recorded using a SPECS NAP-XPS System incorporating the DeviSim NAP reaction cell. The spectrometer is equipped with an $\mathrm{Al} \mathrm{K} \alpha$ monochromatic light source $(\mathrm{h} v=1486.6 \mathrm{eV})$, composed of a SPECS XR50 MF X-ray gun and a $\mu$-FOCUS 600 monochromator, and PHOIBOS 150 NAP 1D-DLD analyser. X-ray gun power was set to $25 \mathrm{~W}(1.68 \mathrm{~mA}$ emission current and $15 \mathrm{kV})$ with a 300 $\mu \mathrm{m}^{2} \mathrm{x}$-ray spot. With this X-ray settings, the intensity of the $\operatorname{Ag}\left(3 \mathrm{~d}_{5 / 2}\right)$ photoemission peak for a $\mathrm{Ag}$ sample, recorded at $10 \mathrm{eV}$ pass energy $(\mathrm{PE})$, was $1 \times 10^{4} \mathrm{cps}$ and the full width at half maximum (FWHM) was $0.60 \mathrm{eV}$. Binding energy calibration was made using $\operatorname{Au}\left(4 \mathrm{f}_{7 / 2}\right)(84.01 \mathrm{eV}), \operatorname{Ag}\left(3 \mathrm{~d}_{5 / 2}\right)(368.20 \mathrm{eV})$, and $\mathrm{Cu}\left(2 \mathrm{p}_{3 / 2}\right)(932.55 \mathrm{eV})$. The survey scans were acquired using $30 \mathrm{eV}$ pass energy, $1 \mathrm{eV}$ step size and 10 seconds $(500 \mathrm{~ms} \times 20$ scans $)$ dwell times. All high resolution spectra $-\mathrm{C}(1 \mathrm{~s}), \mathrm{N}(1 \mathrm{~s})$, and $\mathrm{O}(1 \mathrm{~s})$ - were acquired using $10 \mathrm{eV}$ pass energy, $0.1 \mathrm{eV}$ step size and $75 \operatorname{second}(1 \mathrm{~s} \times$ 75 scans) dwell times. The sample was analysed at an electron take-off angle normal to the surface with respect to the analyser.

The graphene suspension was converted into a slurry and deposited onto carbon based double side sticky tape and dried at $70^{\circ} \mathrm{C}$ for $1 \mathrm{~h}$ in an oven. No significant signs of charging were observed.

\section{Results}

\section{Computational Study}

To complete an earlier study ${ }^{23}$ regarding the $\mathrm{N}(1 \mathrm{~s})$ BEs in graphene, we first provide a broader and accurate study of the different types of single $\mathrm{N}$ atoms in graphene using larger, more realistic, model systems, while keeping the same methodology and basis sets as in the mentioned previous work. Before discussing the results and to avoid possible misunderstandings, it is necessary to point out that, while the comparison between calculated and measured BEs of gas phase molecules is straightforward, a caveat is necessary when the measurements involve condensed matter samples. In the latter case, the measured BEs can only be referred to the vacuum level by taking the 
materials surface work function into account, which is usually avoided by taking an arbitrary reference. For samples containing $\mathrm{C}$ atoms, it is customary to take the $\mathrm{C}(1 \mathrm{~s})$ as a reference as to set it to $\sim 285 \mathrm{eV}$; more precisely we take it as $284.6 \mathrm{eV}$ as justified in the next section describing the XPS measurements. In addition, it is necessary to point out that the experimental values include, obviously, electron correlation and relativistic effects not included in the present HF calculations. The former usually make a small contribution to the $\mathrm{HF} \mathrm{BEs}^{50}$ whereas the latter are essentially atomic in nature, tend to enlarge the HF BEs and also increase with increasing the atomic number. For $\mathrm{C}(1 \mathrm{~s})$ and $\mathrm{N}(1 \mathrm{~s})$ the relativistic contributions increase the HF BEs values by 0.13 and $0.25 \mathrm{eV}$, respectively. ${ }^{21}$ Therefore, to properly compare calculated and measured values one needs to add $0.13 \mathrm{eV}$ to the calculated $\mathrm{C}(1 \mathrm{~s}) \mathrm{BE}$ which, using the same basis set, has been estimated to be $290.25 \pm 0.25 \mathrm{eV}$, and scale all calculated values so that the one digit rounded 290.4 value is set to $284.6 \mathrm{eV}$ as in the experiment. This $5.8 \mathrm{eV}$ energy needs also to be subtracted from the N(1s) HF calculated values once, for consistence, these have been corrected by adding the $0.25 \mathrm{eV}$ contribution of the relativistic effects and rounding it to one digit. In practice, all N(1s) BEs reported in the present work correspond to the raw calculated values scaled down by $5.6 \mathrm{eV}$. Nevertheless, all nonscaled, initial state $(\mathrm{KT})$, final state $(\triangle \mathrm{SCF})$, and relaxation energies, defined as the difference between both values, are reported in the Supplementary Information (SI). The SI also reports the whole set of $\mathrm{KT}$ and $\triangle \mathrm{SCF}$ results for single $\mathrm{N}$ in coronene as computed by treating all $\mathrm{C}$ and $\mathrm{N}$ atoms with the pVTZ basis set, clearly showing that describing the $\mathrm{C}$ atoms with the $6-31 \mathrm{G}^{*}$ basis has an effect on the calculated $\triangle \mathrm{SCF}$ BEs smaller than $0.1 \mathrm{eV}$.

Table 1 reports the full set of calculated results for the different types of single $\mathrm{N}$ in coronene and circumcorone including final state effects and referred to C(1s) at 284.6 $\mathrm{eV}$ as commented above. These set of results show that, in agreement with previous work, ${ }^{23}$ graphitic and Type 2 pyrrolic N (Figure 2) in N-doped graphene exhibit very similar BEs. This is contrary to earlier findings based on calculations including initial state effects where graphitic, pyrrolic, and pyridinic $\mathrm{N}$ exhibit more separated BEs. In fact, this behaviour is also observed in the present set of calculations (see SI). Clearly, final state effects reduce the shift between the BEs for pyrrolic and graphitic types of $\mathrm{N}$ atoms in the graphene layer. The case of pyrrolic $\mathrm{N}$ in five-membered ring (Types 1 and 3) have been considered using the models in Figure S1. The corresponding BEs of 
$399.3 \mathrm{eV}$ and $400.0 \mathrm{eV}$, depending on whether the $\mathrm{N}$ atom is coordinated or not to a hydrogen atom, are still very close to that predicted for graphitic nitrogen. Note also that, except for the Stone-Wales defect, the inclusion of five-membered rings introduces considerable strain in the graphene layer.

From a modelling point of view it is important to point out that values obtained for coronene and circumcoronene models are very similar thus validating the smaller coronene model. In fact, for graphitic and pyrrolic $\mathrm{N}$, the $\triangle \mathrm{SCF}$ calculated $\mathrm{N}(1 \mathrm{~s}) \mathrm{BEs}$ dispersion introduced by using different models is within the typical error quoted for XPS measurements of $0.1 \mathrm{eV} .^{51}$ For the pyridinic cases, the influence of the model is somehow larger with a dispersion value of $0.3 \mathrm{eV}$ well within the resolution of standard equipment. Note also that depending on the immediate environment, two different types of pyridinic $\mathrm{N}$ exist in the circumcoronene model featuring a significant shift in the corresponding $\mathrm{N}(1 \mathrm{~s})$ BEs $(0.6 \mathrm{eV})$, one is bonded to a $\mathrm{C}-\mathrm{H}$ group and a $s p^{2} \mathrm{C}$ atom while the other is bonded to two $s p^{2} \mathrm{C}$ atoms. Nevertheless, the environmentally equivalent pyridinic $\mathrm{N}$ in coronene (A3) and circumcoronene (B5) practically have the same $N(1 \mathrm{~s})$ BEs implying that the influence of the graphene model is within the experimental resolution.

The results discussed so far provide support to the unambiguous assignment of two peaks in the XPS of N-doped graphene. However, as commented in detail in the introduction, most of the experiments reported commonly interpret the XPS spectra of $\mathrm{N}$-doped graphene in terms of three different contributions. To further inspect this possibility, we investigated several other situations including dimers and defects. Table 2 summarizes results from a systematic study of possible N-N dimers on coronene and circumcorone, as depicted in Figure 3. The analysis of results in Table 2 shows some interesting features. First, all the next nearest neighbour graphitic $\mathrm{N}$ in dimers considered situations exhibit $\mathrm{N}(1 \mathrm{~s})$ BEs close to the average of $399.8 \mathrm{eV}$ for a single graphitic $\mathrm{N}$ atom. Hence, next nearest neighbours behave as isolated $\mathrm{N}$ atoms. Second, $\mathrm{N}(1 \mathrm{~s}) \mathrm{BE}$ for graphitic and pyrrolic $\mathrm{N}$ in the dimers are close to each other and larger than that of pyridinic $\mathrm{N}$ by $1.7-1.8 \mathrm{eV}$ as in the isolated $\mathrm{N}$ atom (see Table 1). Third, the $\mathrm{N}(1 \mathrm{~s}) \mathrm{BE}$ of a particular type of $\mathrm{N}$ atom (graphitic, pyrrolic, or pyridinic) in the dimer is close to that of the equivalent type of a single $\mathrm{N}$ atom in the graphene layer although with some influence of the neighbouring $\mathrm{N}$ in the dimer that, for graphitic $\mathrm{N}$, slightly shifts the BEs to larger values, by $0.2 \mathrm{eV}$ only; i.e. from $399.8 \pm 0.1$ (Table 1 ) to $400.0 \pm$ 0.2 (Table 2). A larger effect is found for pyridinic and pyrrolic $\mathrm{N}$ in dimers with BEs 
0.5 and $0.6 \mathrm{eV}$ larger than the corresponding single $\mathrm{N}$ cases. However, the difference is too small to be resolved by standard XPS equipment and, in any case, is likely to contribute to a broadening of the signal spectra. Also, even when this kind of $\mathrm{N}$ dimers could be distinguishable, the BEs do not fit with results from experiments where the deconvolution of the XPS spectra is made on the basis of three different peaks which appear to have shifts of around $1 \mathrm{eV}$ with respect to each other. ${ }^{13-17}$

In a final attempt to look for an alternative situation for $\mathrm{N}$ in the graphene layer, four main defects have been studied (Figure 4). The first involves three $\mathrm{N}$ around a double carbon vacancy where one $\mathrm{N}$ is added and edge carbons are substituted by $\mathrm{N}$ atoms (Figure 4A); it represents a low probability situation and is considered for completeness only. This type of defect involves pyrrolic and pyridinic $\mathrm{N}$ atoms, for the latter the calculated $\mathrm{N}(1 \mathrm{~s}) \mathrm{BE}$ is $397.2 \mathrm{eV}$, just $0.6 \mathrm{eV}$ below the single pyridinic case. The second defect involves also a double carbon vacancy with a $\mathrm{H}$ terminated pyrrolic $\mathrm{N}$ (Figure 4B) whereas the third is the same but with the two edge $\mathrm{C}$ atoms also terminated by hydrogen atoms. In these types of defects with pyrrolic $\mathrm{N}$, the calculated $\mathrm{N}(1 \mathrm{~s})$ BEs are 399.1 and $399.3 \mathrm{eV}$, close enough to the BE of the single pyrrolic $\mathrm{N}$. Finally, for $\mathrm{N}$ in a Stone-Wales defect ${ }^{41}$ the calculated $\mathrm{N}(1 \mathrm{~s}) \mathrm{BE}$ is $399.9 \mathrm{eV}$, very close to the value for single graphitic $\mathrm{N}$ atoms.

From the complete set of calculated results it appears that graphitic and pyrrolic $\mathrm{N}$ atoms in graphene exhibit very close $\mathrm{N}(1 \mathrm{~s})$ BEs and are both rather separated from the corresponding value for pyridinic N. Situations where two N atoms are separated by a $\mathrm{C}$ atom can be considered as single $\mathrm{N}$ atoms whereas for each type of $\mathrm{N}$ in a dimer, the calculated N(1s) BEs is just slightly shifted. At this point, one may wonder whether the common interpretation based in deconvoluting the N(1s) XPS into three contributions holds. This aspect is investigated in detail in the next section.

\section{Experimental Study}

The survey spectrum in Figure S2 shows the N-doped graphene characteristic peaks: carbon, nitrogen, oxygen, and a small contribution from silicon that could come from the grease or glass etching during the synthetic procedure. The calculated nitrogen percentage is $2.0 \%$ at. in the surface near region, using Schofield sensitivity factors.

Casa XPS was used for data interpretation of the recorded N(1s) spectra of Ndoped graphene samples prepared as described above. Shirley or two-point linear background subtractions were employed depending on background shape. The N(1s) 
peak for graphitic nitrogen was fitted using a $\mathrm{A}(0.3,0.4,50) \mathrm{GL}(30)$ asymmetric function characteristic of conductive moieties and, similarly to the carbon signal for graphite. The rest of the peaks were fitted using GL(30) lineshapes, which involve a combination of a Gaussian $(70 \%)$ and Lorentzian $(30 \%)$. The atomic percentages were calculated from the peak areas in the acquired high-resolution spectra. Casa XPS relative sensitivity factors based on Scofield cross-sections were used for atomic percentage quantification analysis (where the relative sensitivity factor of $C(1 \mathrm{~s})$ is set to 1.000). The fitted contribution of C-C graphitic like carbon in the $\mathrm{C}(1 \mathrm{~s})$ signal was measured at $284.6 \mathrm{eV}$ (see $\mathrm{C}(1 \mathrm{~s})$ spectrum $\mathrm{S} 3$ ). This is also the reason to refer all calculated values in the previous section and Tables 1 and 2 to this value.

The N(1s) spectrum acquired is similar to the one acquired by Pendashteh et $a l{ }^{49}$ for a similar sample. Two main fitting models, denoted A and B in Table 3, are used to fit the $\mathrm{N}(1 \mathrm{~s})$ spectrum. The first one uses two main peaks, one for pyridinic $\mathrm{N}$ atoms and one involving both graphitic $\mathrm{N}$ and pyrrolic $\mathrm{N}$ signals, whereas the second model uses three main peaks (one for each type of structurally different $\mathrm{N}$ atoms). In the fittings, as we have no previous information on individual contributions, we have constrained the fitting for all these peaks to have the same full width at half maximum (FWHM). We also include a peak to take into account for pyridine based nitrogen oxide like impurities (forced at $\mathrm{BE}>401.5 \mathrm{eV}$ ) and one for the characteristic shake up signal characteristic of materials containing double bonded nitrogen atoms. ${ }^{4}$

The N(1s) spectrum featuring the two different fitting models are shown in Figure 6 and the results summarized in Table 3. The two fittings are both mathematically correct and offer some interesting insight. First, the separation between pyrrolic and graphitic peaks $(0.5 \mathrm{eV})$ is much smaller than the FWHM of the peaks $(1.5$ $\mathrm{eV}$ ). Two peaks can only be fully resolved if they are separated by more than the FWHM. Therefore, without being able to analyse both contributions separately, it is not possible to extract the exact BE position and FWHM of these peaks and consequently, it is not possible to separate the two contributions with physical meaning. As a consequence, separating these two peaks using subjective BE positions and FWHM could give rise to the calculation of erroneous atomic relative percentages. In fact, the fitting model using three peaks (Figure 6) predicts an exceedingly large number of pyrrolic $\mathrm{N}$ which is hardly acceptable. These observations suggest that considering a two peaks fitting model will be more appropriate, as using three peaks can lead to illsupported physical information. Indeed, the fit to two peaks leads to calculated BE 
values of $398.4 \mathrm{eV}$ for pyridinic $\mathrm{N}$, very close to the calculated average value for the different types of pyridinic N. Using also this model, the predicted value for graphitic/pyrrolic $\mathrm{N}(399.7 \mathrm{eV})$ matches the average calculated binding energy value for these situations.

\section{Conclusions}

A series of $\mathrm{N}$-doped graphene models including isolated nitrogen atoms, dimers, and defects have been considered to shed light on the interpretation of N(1s) XPS in these systems. For each situation the $\mathrm{N}(1 \mathrm{~s}) \mathrm{BE}$ has been calculated from $\Delta \mathrm{SCF}$ calculations using the Hartree-Fock method using sufficiently large basis sets, a procedure that includes initial and final state contributions to the BEs with mean errors below $0.5 \mathrm{eV}$ for the BEs and below $0.1 \mathrm{eV}$ for the BE shifts. Therefore, it is expected that the differences between the BEs for the different types of $\mathrm{N}$ in the different models are accurate enough so as to provide a sound benchmark. All results consistently show that, once final state effects are taken into account, $\mathrm{N}(1 \mathrm{~s})$ for graphitic and pyrrolic $\mathrm{N}$ are too close to be distinguishable by XPS which calls for a revision of the common assignment. This is not the case for pyridinic $\mathrm{N}$ which exhibits a significantly different $\mathrm{BE}$ and the corresponding assignment is well supported by the present ab initio calculations.

The conclusions from the theoretical study are supported by new XPS experiments on $\mathrm{N}$-doped graphene samples where the $\mathrm{N}(1 \mathrm{~s})$ broad feature has been fitted to two or three peaks. Both fits are meaningful but, on the one hand, the three peaks fit leads to assignments for graphitic and pyrrolic $\mathrm{N}$ that are too close in BE to justify the fitting of this part of the experimental signal with two separate peaks and, on the other hand, the fit to two peaks is consistent with the predictions from theory. Thus, the present work strongly suggests that the interpretation of the XPS spectra of N-doped graphene has to be revised by considering that graphitic and pyrrolic $\mathrm{N}$ cannot be distinguished with enough accuracy to provide physical meaning. 


\section{Acknowledgements}

This research was supported by the Spanish MINECO/FEDER CTQ2015-64618-R, ENE2016-79608-C2-1-R (RaPHUEL) and ENE2016-82025-REDT (FOTOFUEL) projects, by Generalitat de Catalunya (grants 2017SGR13 and XRQTC). F.V. thanks MINECO for a postdoctoral Ramón y Cajal (RyC) research contract (RYC-201210129) and F.I. acknowledges additional support from the 2015 ICREA Academia Award for Excellence in University Research. Financial support from Spanish MICINN through the Excellence Maria de Maeztu program (grant MDM-2017-0767) is also fully acknowledged.

\section{Supporting Information for Publication}

Initial and final CLBEs for $\mathrm{N}$ single and dimer configurations using HF, comparative between basis sets, models for Type 1 and Type 3 pyrrolic N, spectrum of the N-doped graphene samples, XPS spectra of C(1s) for N-doped graphene. 
Table 1. Calculated N(1s) BEs for different types of single nitrogen atoms in N-doped modelled by coronene and circumcoronene as shown in Figure 1. All values have been obtained at Hartree-Fock level within the $\triangle \mathrm{SCF}$ approach. Values are given relative to $\mathrm{C}(1 \mathrm{~s})$ in these systems taken as $284.6 \mathrm{eV}$ and after adding the atomic relativistic contribution to both $\mathrm{C}(1 \mathrm{~s})$ and $\mathrm{N}(1 \mathrm{~s})$ BEs. $\Delta$ stands for the average for each type of $\mathrm{N}$ and error bars correspond to the dispersion. All values are given in $\mathrm{eV}$.

\begin{tabular}{|c|c|c|c|}
\hline \multicolumn{2}{|c|}{ N type } & $\mathrm{N}(1 \mathrm{~s}) \mathrm{BE}$ & $\Delta$ \\
\hline \multirow{6}{*}{ Graphitic } & A1 & 399.8 & \multirow{6}{*}{$399.8 \pm 0.1$} \\
\hline & $\mathrm{A} 2$ & 399.9 & \\
\hline & B1 & 399.8 & \\
\hline & B2 & 399.7 & \\
\hline & B3 & 399.8 & \\
\hline & B4 & 399.8 & \\
\hline \multirow{3}{*}{ Pyrrolic } & A3 & 399.6 & \multirow{3}{*}{$399.5 \pm 0.1$} \\
\hline & B5 & 399.5 & \\
\hline & B6 & 399.5 & \\
\hline \multirow{3}{*}{ Pyridinic } & A3 & 398.1 & \multirow{3}{*}{$397.8 \pm 0.3$} \\
\hline & B5 & 397.9 & \\
\hline & B6 & 397.3 & \\
\hline
\end{tabular}


Table 2. Calculated N(1s) BEs for different types of N-N dimers atoms in coronene and circumcoronene as shown in Figure 3. Leftmost column indicated the type core ionized whereas the next column indicates the type of $\mathrm{N}$ neighbour and its environment (Figure 1). All values are in $\mathrm{eV}$ and obtained as explained in Table 1.

\begin{tabular}{|c|c|c|c|}
\hline \multicolumn{2}{|c|}{$\mathbf{N}-\mathbf{N}$ dimers } & $\mathbf{N}(1 s) \mathbf{B E}$ & $\Delta$ \\
\hline \multirow{13}{*}{ Graphitic } & A1-A4 (graphitic) & 400.0 & \multirow{13}{*}{$400.0 \pm 0.2$} \\
\hline & A1-A5 $(n, n, n)$ & 399.7 & \\
\hline & A2-A3 (pyridinic) & 400.3 & \\
\hline & B1-B8 (graphitic) & 400.1 & \\
\hline & B1-B9 $(n, n, n)$ & 400.0 & \\
\hline & B1-B2 (graphitic) & 399.8 & \\
\hline & B2-B3 (graphitic) & 400.0 & \\
\hline & B2-B10 (n,n,n) & 399.6 & \\
\hline & B3-B4 (graphitic) & 400.1 & \\
\hline & B4-B6 (pyrrolic) & 400.2 & \\
\hline & B4-B6 (pyridinic) & 400.2 & \\
\hline & B4-B5 (pyrrolic) & 400.5 & \\
\hline & B4-B5 (pyridinic) & 400.1 & \\
\hline \multirow{5}{*}{ Pyrrolic } & A3-A6 (pyridinic) & 400.2 & \multirow{5}{*}{$400.1 \pm 0.1$} \\
\hline & B6-B4 (graphitic) & 400.1 & \\
\hline & B5-B4 (graphitic) & 399.9 & \\
\hline & B5-B7 (pyridinic) & 400.1 & \\
\hline & B5-B7 (pyrrolic) & 400.1 & \\
\hline \multirow{7}{*}{ Pyridinic } & A3-A6 (pyridinic) & 399.1 & \multirow{7}{*}{$398.3 \pm 0.5$} \\
\hline & A3-A6 (pyrrolic) & 398.4 & \\
\hline & A3-A2 (graphitic) & 398.2 & \\
\hline & B6-B4 (graphitic) & 397.7 & \\
\hline & B5-B4 (graphitic) & 397.7 & \\
\hline & B5-B7 (pyridinic) & 398.9 & \\
\hline & B5-B7 (pyrrolic) & 398.2 & \\
\hline
\end{tabular}


Table 3. XPS N(1s) data collected on N-doped graphene for each proposed model. (A) Considering two types of distinct $\mathrm{N}$ (pyridine and graphitic/pyrrolic) and (B) by using three types of distinct $\mathrm{N}$ (pyridine, graphitic and pyrrolic). N-oxide and Shake-up signals are also included.

\begin{tabular}{ccccc}
\hline XPS Model & N Assignment & BE $(\mathbf{e V})^{\boldsymbol{a}}$ & Atomic $^{\boldsymbol{\%}^{\boldsymbol{b}}}$ & FWHM $^{\boldsymbol{c}}$ \\
\hline \multirow{3}{*}{ A } & Pyridinic & 398.4 & 41.8 & 1.5 \\
& Graphitic/Pyrrolic & 399.7 & 48.9 & 1.5 \\
& N-oxide & 401.5 & 5.6 & 2 \\
& Shake up & 404.1 & 3.7 & 2.5 \\
\hline \multirow{2}{*}{ B } & Pyridinic & 398.4 & 37.9 & 1.5 \\
& Pyrrolic & 399.5 & 27.2 & 1.5 \\
& Graphitic & 400.0 & 23.3 & 1.5 \\
& N-oxide & 401.5 & 8.2 & 2.0 \\
& Shake up & 404.1 & 3.4 & 2.5 \\
\hline
\end{tabular}

${ }^{a}$ Binding Energy, BE (in eV); ${ }^{b}$ Atomic concentration (\%). ${ }^{c}$ Full Width at Half Maximum (FWHM) 
Figure 1. Coronene (A) and circumcoronene (B) molecules taken as models for graphene flakes. Grey and white spheres denote $\mathrm{C}$ and $\mathrm{H}$ atoms, respectively. Red spheres denote symmetry distinct $\mathrm{C}$ atoms where $\mathrm{C}$ or $\mathrm{C}-\mathrm{H}$ are substituted by $\mathrm{N}$ (see text). Labels denote the different explored possibilities.

A)

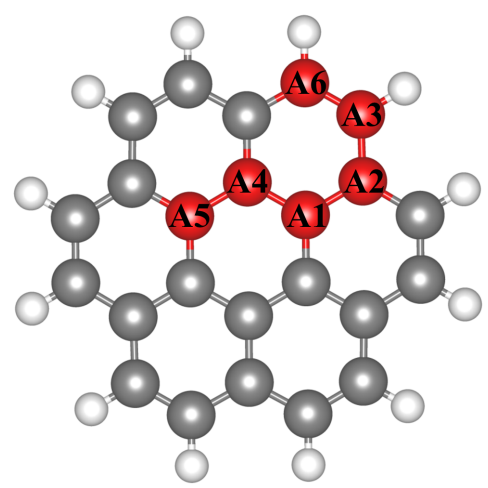

B)

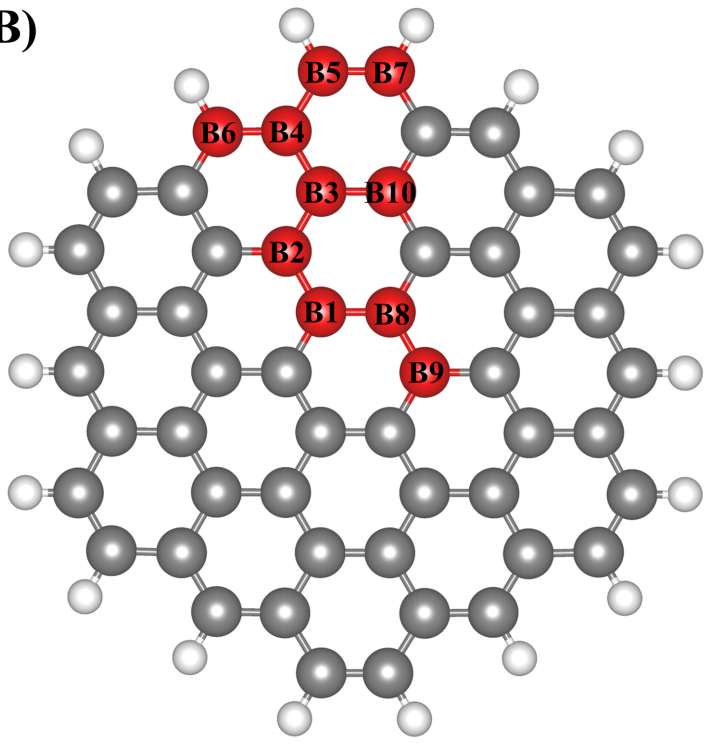


Figure 2. Graphitic (A), Pyridinic (B), Type 2 pyrrolic (C), Type 3 pyrrolic (D), and Type 1 pyrrolic $\mathrm{N}$ (E). Grey, white and red stand for $\mathrm{C}, \mathrm{H}$ and $\mathrm{N}$ atoms, respectively.

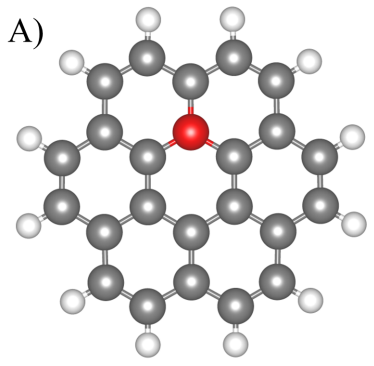

D)

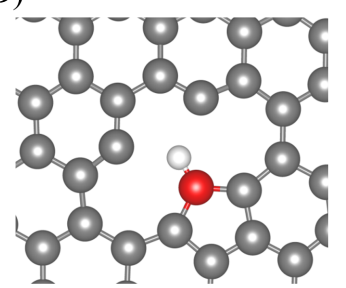

B)

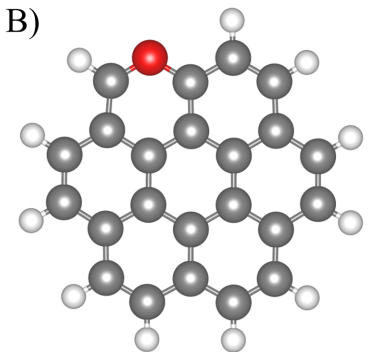

E)

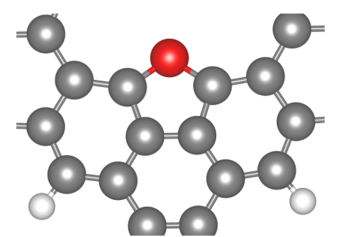

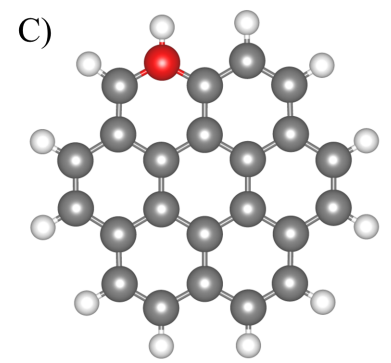


Figure 3. Nearest neighbour (nn) graphitic $\mathrm{N}$ dimer (A), next nearest neighbour (nnn) graphitic $\mathrm{N}$ dimer (B), nearest neighbour $\mathrm{N}$ dimer involving graphitic and pyridinic $\mathrm{N}$ (C) and nearest neighbour $\mathrm{N}$ dimer involving graphitic and pyrrolic N. Colour code as in Figure 2.

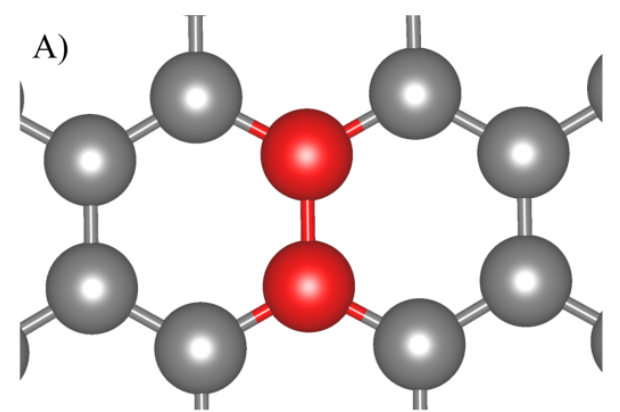

C)

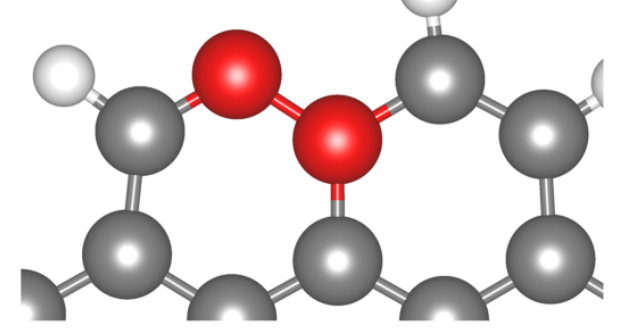

B)

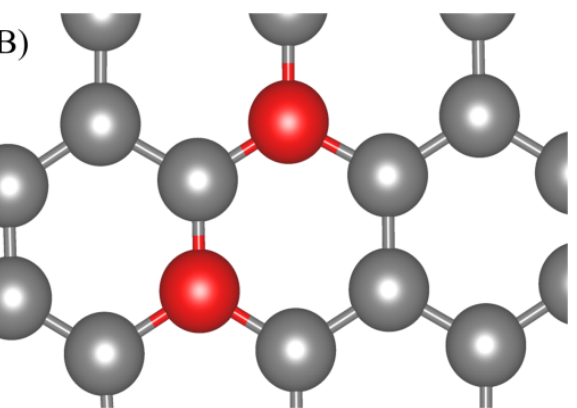

D)

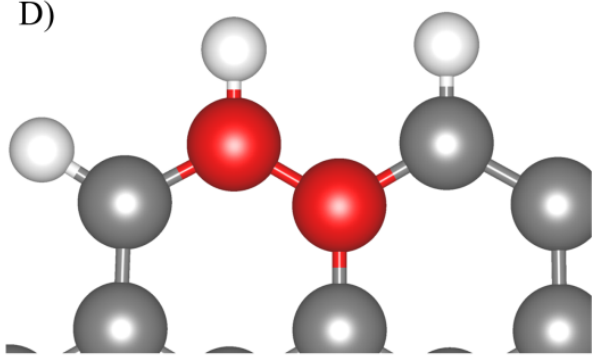


Figure 4. Several possibilities of $\mathrm{N}$ at a double $\mathrm{C}$ vacancy defect. $\mathrm{N}$ atoms are added while keeping the $2 \mathrm{C}$ vacancy (A), a $2 \mathrm{C}$ vacancy defect with one added $\mathrm{N}-\mathrm{H}$ group (B), a $2 \mathrm{C}$ vacancy defect, adding one $\mathrm{N}-\mathrm{H}$ group and with $\mathrm{H}$ saturation of edge $\mathrm{C}$ atoms (C). Note that A contains 2 pyridinic and one Type 1 pyrrolic N. Both B and C display Type 3 pyrrolic N. Colour code as in Figure 2.
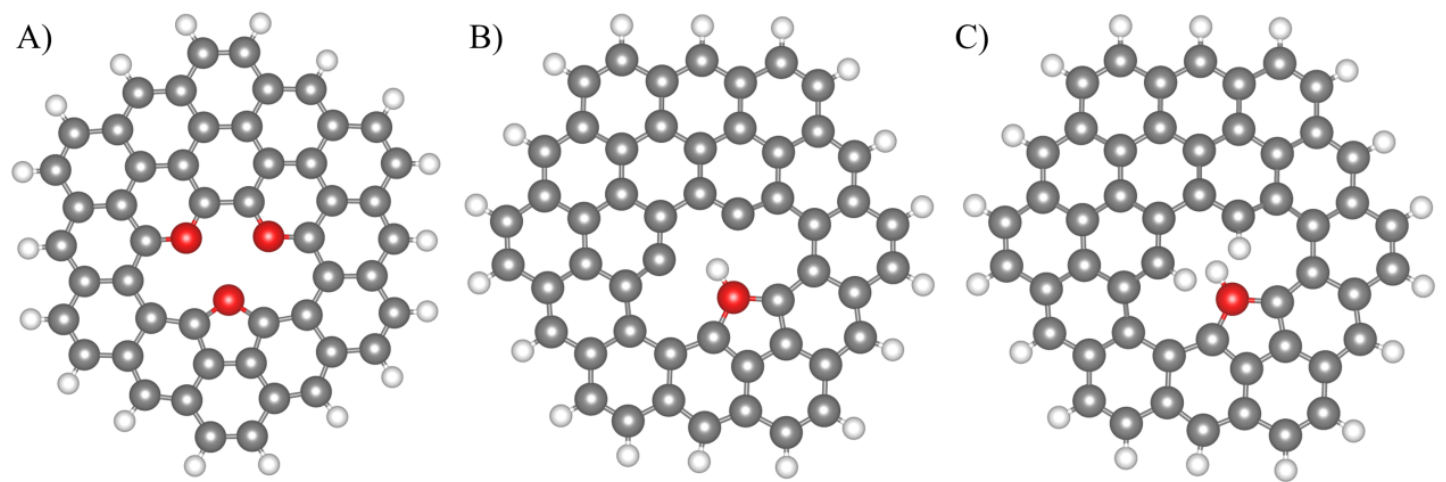
Figure 5. Schematic representation of the formation of a Stone-Wales defect (A). A Stone-Wales defect where one $\mathrm{C}$ atom has been substituted by a $\mathrm{N}$ atom (B).

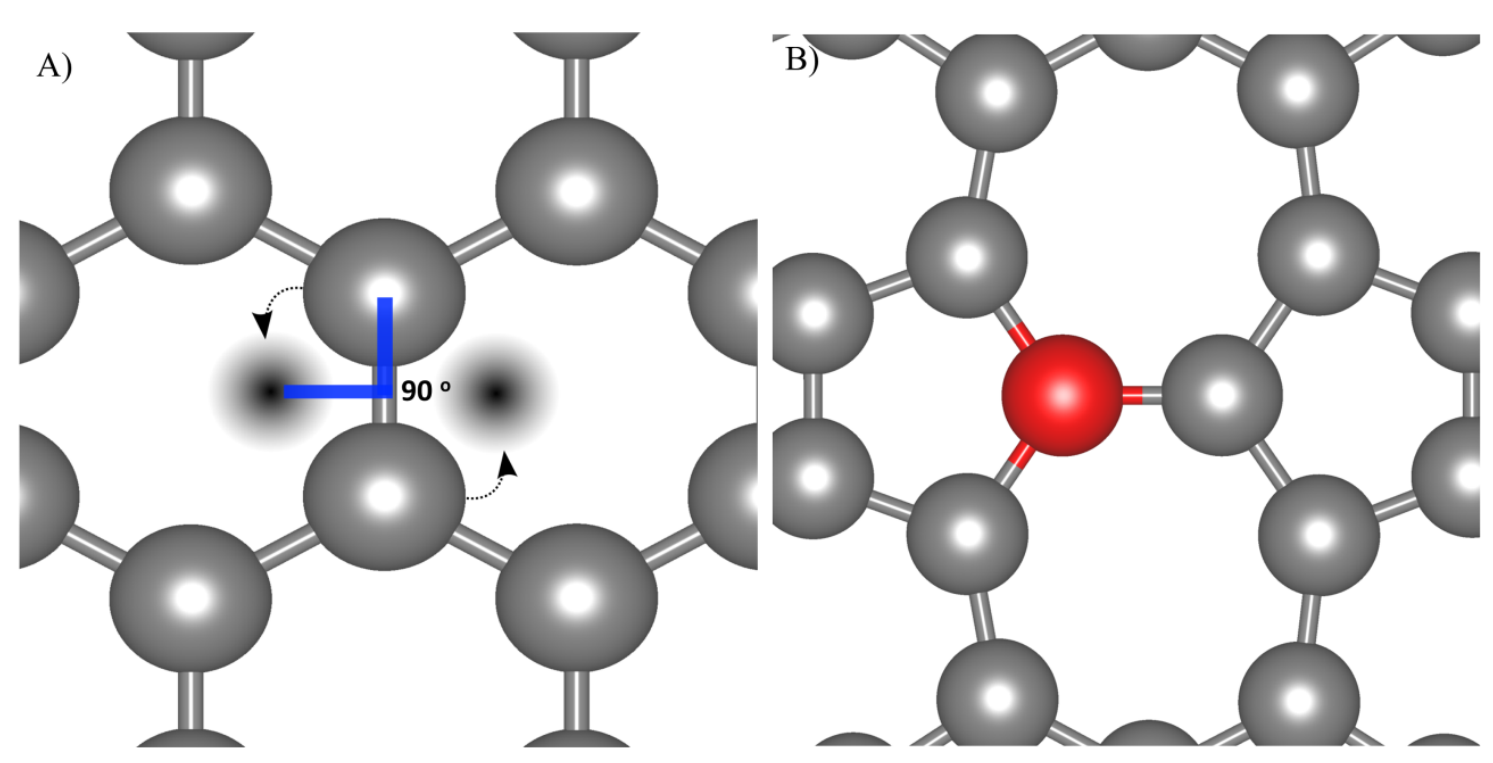


Figure 6. N(1s) XPS of N-doped graphene fitted by (A) considering two types of distinct $\mathrm{N}$ (pyridine and graphitic/pyrrolic) and (B) by considering three types of distinct $\mathrm{N}$ (pyridine, graphitic and pyrrolic). In both cases signals ascribed to N-O and shake up features are also observed.
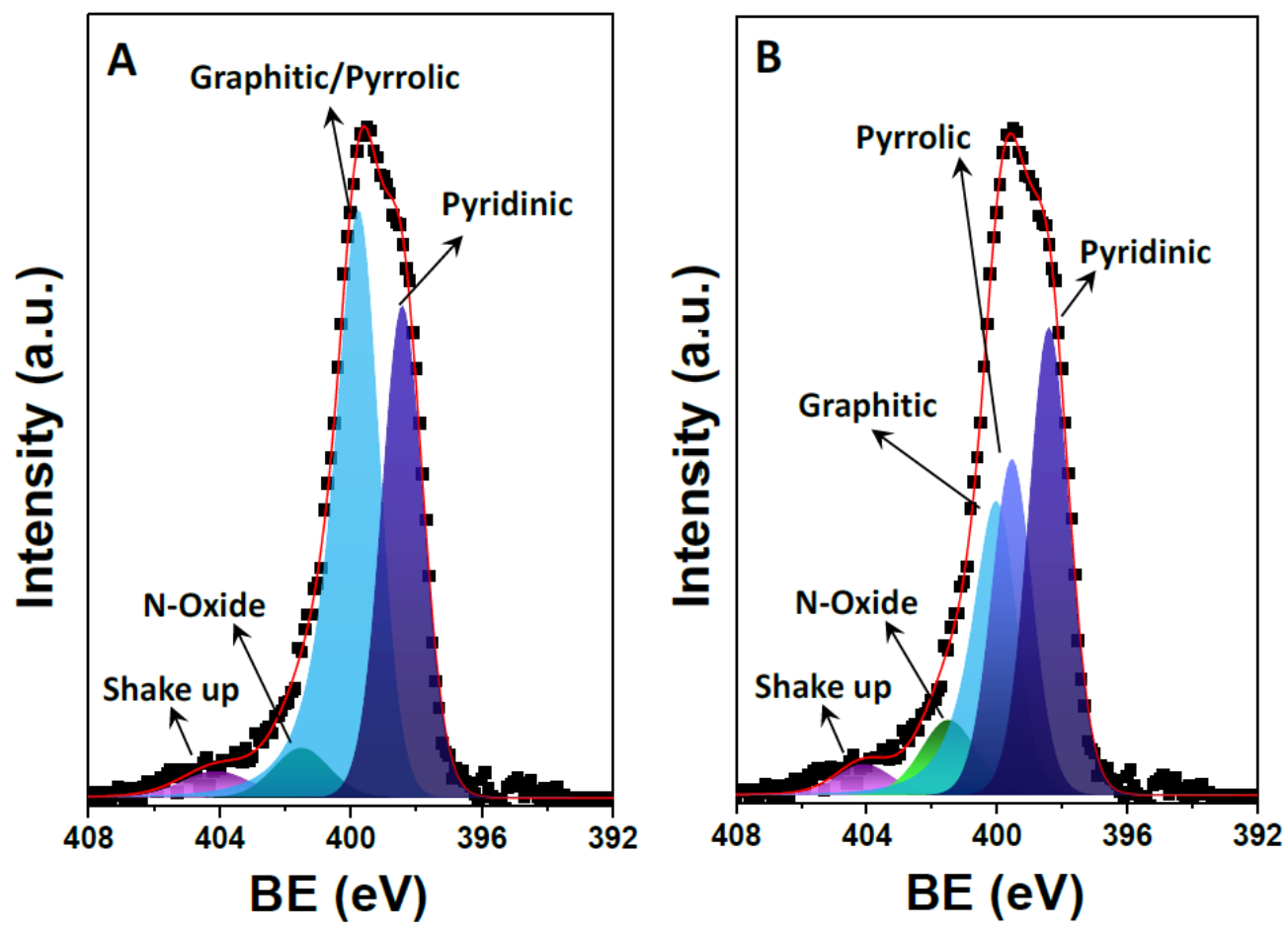


\section{References}

${ }^{1}$ Novoselov, K. S.; Geim, A. K.; Morozov, S. V.; Jiang, D.; Zhang, Y.; Dubonos, S. V. Electric Field Effect in Atomically Thin Carbon Films. Science 2004, 306, 666-669.

${ }^{2}$ Geim, A. K.; Novoselov, K. S. The Rise of Graphene. Nature Mater. 2007, 6, 183191.

${ }^{3}$ Neto, A. H. C.; Guinea, F.; Peres, N. M. R.; Novoselov, K. S.; Geim, A. K. The Electronic Properties of Graphene. Rev. Mod. Phys, 2009, 81, 109-162.

${ }^{4}$ Allen, M. J.; Kaner, R. B.; Tung, V. C. Honeycomb Carbon: a Review of Graphene. Chem. Rev. 2010, 110, 132-145.

${ }^{5}$ Wang, X. R.; Li, X. L.; Zhang, L.; Yoon, Y.; Weber, P. K.; Wang, H. L. N-doping of Graphene through Electrothermal Reactions with Ammonia. Science 2009, 324, 768771.

${ }^{6}$ Saha, S. K.; Ahirwal, A.; Panchakarla, L. S.; Waghmare, U. V.; Panchokarla, L. S.; Subrahmanyam, K. S.; Govindaraj, A.; Rao, C. N. R.; Saha, S. K.; Krishnamurthy, H. R. Synthesis, Structure, and Properties of Boron- and Nitrogen-Doped Graphene. Adv. Mater. 2009, 21, 4726-4730.

${ }^{7}$ Sokolowski, E.; Nordling, C.; Siegbahn, K. Chemical Shift Effect in Inner Electronic Levels of Cu Due to Oxidation. Phys. Rev., 1958, 110, 776.

${ }^{8}$ Bagus, P. S.; Illas, F.; Pacchioni, G.; Parmigiani, F. Mechanisms Responsible for Chemical Shifts of Core-Level Binding Energies and their Relationship to Chemical Bonding. J. Electron Spectros. Relat. Phenomena 1999, 100, 215-236.

${ }^{9}$ Delesma, F. A.; Van den Bossche, M.; Grönbeck, H.; Calaminici, P.; Köster, A. M.; Pettersson, L. G.M. A Chemical View on $\mathrm{X}$ ray Photoelectron Spectroscopy: the ESCA Molecule and Surface to Bulk XPS Shifts. ChemPhysChem, 2018, 19, 169174.

${ }^{10}$ Pels, J.R.; Kapteijn, F.; Moulijn, J.A.; Zhu, Q.; Thomas, K.M. Evolution of Nitrogen Functionalities in Carbonaceous Materials during Pyrolysis. Carbon 1995, 33, 16411653.

${ }^{11}$ Casanovas, J.; Ricart, J. M.; Rubio, J.; Illas, F.; Jimenez Mateos, J.M. Origin of the Large $\mathrm{N}$ is Binding Energy in X-ray Photoelectron Spectra of Calcined Carbonaceous Materials. JACS 1996, 118, 8071-8076.

12 Bundaleska, N.; Henriques, J.; Abrashev, M.; Botelho do Rego, A. M.; Ferraria, A. M.; Almeida, A.; Dias, F. M.; Valcheva, E.; Arnaudov, B.; Upadhyay, K. K.; et al. 
Large-Scale Synthesis of Free-Standing N-Doped Graphene Using Microwave Plasma. Sci. Rep. 2018. 8, 12595.

${ }^{13}$ Mansour, R.; Reza, N. M.; Mahmoud, P.; Hasuck, K. Facile Enhancement of the Active Catalytic Sites of N-Doped Graphene as a High Performance Metal-Free Electrocatalyst for Oxygen Reduction Reaction. Appl. Surf. Sci. 2018, 447, 182-190.

${ }^{14}$ Cao, Y.; Si, W.; Li, J.; Wang, F.; Zhang, Y.; Hao, Q.; Lei, W.; Xia, X. NitrogenDoped Graphene: Effect of Graphitic-N on the Electrochemical Sensing Properties Towards Acetaminophen. FlatChem 2018, 9, 1-7.

${ }^{15}$ Dai, S.; Zhao, B.; Hu, H.; Zhang, Q.; Chen, D.; Qu, C.; Dang, D.; Liu, M.; Liu, Z.;

Zeng, J. A High-Performance Supercapacitor Electrode Based on N-Doped Porous Graphene. J. Power Sources 2018, 387, 43-48.

${ }^{16}$ Chen, X.; Zhang, G.; Shi, L.; Pan, S.; Liu, W.; Pan, H. Au/ZnO Hybrid Nanocatalysts Impregnated in N-Doped Graphene for Simultaneous Determination of Ascorbic Acid, Acetaminophen and Dopamine. Mater. Sci. Eng. C 2016, 65, 80-89.

${ }^{17}$ Wang, C. Y.; Shao, Y.; Matson, D. W.; Li, J.; Lin, Y. Nitrogen-Doped Graphene and its Application in Electrochemical Biosensing. ACS Nano 2010, 4, 1790-1798.

${ }^{18}$ Xu, H.; Ma, L.; Jin, Z. Nitrogen-Doped Graphene: Synthesis, Characterizations and Energy Applications. J. Energy Chem. 2018, 27, 146-160.

${ }^{19}$ Lai, L.; Potts, J. R.; Zhan, D.; Wang, L.; Poh, C. K.; Tang, C.; Gong, H.; Shen, Z.; Lin, J.; Ruoff, R. S. Exploration of the Active Center Structure of Nitrogen-Doped Graphene-Based Catalysts for Oxygen Reduction Reaction. Energy \& Environ. Sci. 2012, 5, 7936-7942.

${ }^{20}$ Bagus, P. S.; Ilton, E. S.; Nelin, C. J. The Interpretation of XPS Spectra: Insights into Materials Properties. Surf. Sci. Rep. 2013, 68, 273-304.

21 Bellafont, N. P.; Viñes, F.; Illas, F. Performance of the TPSS Functional on Predicting Core Level Binding Energies of Main Group Elements Containing Molecules: A Good Choice for Molecules Adsorbed on Metal Surfaces. J. Chem. Theory Comput. 2015, 12, 324-331.

${ }^{22}$ Bellafont, N. P.; Viñes, F.; Hieringer, W.; Illas, F. Predicting Core Level Binding Energies Shifts: Suitability of the Projector Augmented Wave Approach as Implemented in VASP. J. Comput. Chem. 2017, 38, 518-522. 
${ }^{23}$ Bellafont, N. P.; Mañeru, D. R.; Illas, F. Identifying Atomic Sites in N-Doped Pristine and Defective Graphene from Ab Initio Core Level Binding Energies. Carbon 2014, 76, 155-164.

24 Inagaki, M.; Toyoda, M.; Soneda, Y.; Morishita, T. Nitrogen-doped Carbon Materials. Carbon 2018, 132, 104-140.

${ }^{25}$ Zhao, W.; Hofert, O.; Gotterbarm, K.; Zhu, J. F.; Papp, C.; Steinruck, H. -P. Production of Nitrogen-Doped Graphene by Low-Energy Nitrogen Implantation $J$. Phys. Chem. C 2012, 116, 5062-5066

${ }^{26}$ Koch, R. J.; Weser, M.; Zhao, W.; Vines, F.; Gotterbarm, K.; Kozlov, S. M.; Hofert, O.; Ostler, M.; Papp, C.; Gebhardt, J.; et al. Growth and Electronic Structure of Nitrogen-doped Graphene on Ni(111). Phys. Rev. B 2012, 86, 075401.

${ }^{27}$ Jung, R.; Cheong, J.-K. Investigation of the Dependence of the Chemical States of the Graphene Surface on $\mathrm{N}_{2}$ Plasma Treatment. J. Korean Phys. Society 2012, 60, 933-936. ${ }^{28}$ Li, T.; Guo, R.; Luo, Y.; Li, F.; Liu, Z.; Meng, L.; Yang, Z.; Luo, H.; Wan, Y. Innovative N-Doped Graphene-Coated $\mathrm{WS}_{2}$ Nanosheets on Graphene Hollow Spheres Anode with Double-Sided Protective Structure for Li-Ion Storage. Electrochim. Acta 2018, 290, 128-141.

${ }^{29}$ Luque-Centeno, J. M.; Martínez-Huerta, M. V.; Sebastián, D.; Lemes, G.; Pastor, E.; Lázaro, M. J. Bifunctional N-doped Graphene Ti and Co Nanocomposites for the Oxygen Reduction and Evolution Reactions. Renew. Energ. 2018, 125, 182-192.

${ }^{30}$ Lobinsky, A. A.; Tolstoy, V. P. Synthesis of CoAl-LDH Nanosheets and N-Doped Graphene Nanocomposite Via Successive Ionic Layer Deposition Method and Study of their Electrocatalytic Properties for Hydrogen Evolution in Alkaline Media. J. Solid State Chem. 2019, 270, 156-161.

${ }^{31}$ Li, Y.; Wen H.; Yang, J.; Zhou, Y.; Cheng, X. Boosting Oxygen Reduction Catalysis with N, F, and S Tri-Doped Porous Graphene: Tertiary N-Precursors Regulates the Constitution of Catalytic Active Sites. Carbon 2019, 142, 1-12.

${ }^{32}$ Beamson, G.; Briggs, D. High Resolution XPS of Organic Polymers. The Scienta ESCA300 Database. John Wiley \& Sons. 1992.

${ }^{33}$ Carraro G.; Celasco, E.; Smerieri, M.; Savio, L.; Bracco, G.; Rocca, M.; Vattuone, L. Chemisorption of CO on N-Doped Graphene on Ni(111). Appl. Surf. Sci. 2018, 428, 775-780. 
${ }^{34}$ Hu, Q.; Liu, X.; Zhu, B.; Fan, L.; Chai, X.; Zhang, Q.; Liu, J.; He, C.; Lin, Z. Crafting $\mathrm{MoC}_{2}$-doped Bimetallic Alloy Nanoparticles Encapsulated within N-Doped Graphene as Roust Bifunctional Electrocalalysts for Overall Wáter Splitting. Nano Energy 2018, 50, 212-219.

${ }^{35}$ Du, Y.; Liu, L.; Xiang, Y.; Zhang, Q. Enhanced Electrochemical Capacitance and Oil-Absorbability of N-Doped Graphene Aerogel by Using Amino-Functionalized Silica as Template and Doping Agent. J. Power Sources 2018, 379, 240-248.

${ }^{36}$ Ni, S.; W. Wang, D. H.; Bao, Y.; Han, D.; Wang, H.; Niu, L.; Han, F. Innovations Upon Antioxidant Capacity Evaluation for Cosmetics: A Photoelectrochemical Sensor Exploitation Based on N-Doped Graphene/TiO 2 Nanocomposite. Sens. Actuator BChem. 2018, 259, 963-971.

${ }^{37}$ Wang, H.; Maiyalagan, T.; Wang, X. Review on Recent Progress in Nitrogen-Doped Graphene: Synthesis, Characterization, and its Potential Applications. ACS catal. 2012, $2,781-794$.

${ }^{38}$ Li, X. F.; Lian, K. Y.; Liu, L.; Wu, Y.; Qiu, Q.; Jiang, J.; Deng, M.; Luo, Y. Unraveling the Formation Mechanism of Graphitic Nitrogen-Doping in Thermally Treated Graphene with Ammonia. Sci. Rep. 2016, 6, 23495.

${ }^{39}$ Hassan, F. M.; Chabot, V.; Li, J.; Kim, B. K.; Sandoval, L. R.; Yu, A. PyrrolicStructure Enriched Nitrogen Doped Graphene for Highly Efficient Next Generation Supercapacitors. J. Mater. Chem. A 2013, 1, 2904-2912.

${ }^{40}$ Fomine, S.; Torres, A. Electronic Structure of Graphene Nanoribbons Doped with Nitrogen Atoms: A Theoretical Insight. Phys. Chem. Chem. Phys. 2015, 17, 1060810614.

${ }^{41}$ Ma, J.; Alfè, D.; Michaelides, A.; Wang, E. Stone-Wales Defects in Graphene and other Planar sp2-Bonded Materials. Phys. Rev. B 2009, 80, 33407.

${ }^{42}$ Ditchfie R, Hehre WJ, Pople JA. Self-Consistent Molecular-Orbital Methods. IX. Extended Gaussian-Type Basis for Molecular-Orbital Studies of Organic Molecules. $J$. Chem. Phys. 1971, 54, 724-728.

${ }^{43}$ Schäfer, A.; Horn, H.; Ahlrichs, R. Fully Optimized Contracted Gaussian Basis Sets for Atoms Li to Kr. J. Chem. Phys. 1992, 97, 2571-2577.

44 Mcclean, A. D.; Chandler, G. S.; Contracted Gaussian-Basis Sets for Molecular Calculations .1. $2^{\text {nd }}$ Row Atoms, $Z=11-18 . J$. Chem. Phys. 1980, 72, 5639-5648. 
45 Bühl, M.; Kabrede, H. Geometries of Transition-Metal Complexes from DensityFunctional Theory. J. Chem. Theory Comput. 2006, 2, 1282-1290.

${ }^{46}$ Viñes, F.; Sousa, C.; Illas, F. On the Prediction of Core Level Binding Energies in Molecules, Surfaces and Solids. Phys. Chem. Chem. Phys. 2018, 20, 8403-8410

${ }^{47}$ Schimdt, M. W.; Baldridge, K. K.; Boatz, J. A.; Elbert, S. T.; Gordon, M. S.; Jensen, J.H.; Koseki, S.; Matsunaga, N.; Nguyen, K. A.; Su, S.; et al. General Atomic and Molecular Electronic Structure System. J. Comput. Chem. 1993, 14, 1347-1363.

${ }^{48}$ Bagus, P. S. Self-Consistent-Field Wave Functions for Hole States of Some Ne-Like and Ar-Like Ions. Phys. Rev. 1965, 139, A629-A634.

${ }^{49}$ Pendashteh, A.; Palma, J.; Anderson, M.; Marcilla, R. NiCoMnO 4 Nanoparticles on N-doped Graphene: Highly Efficient Bifunctional Electrocatalyst for Oxygen Reduction/Evolution Reactions. Appl. Catal. B-Environ. 2017, 201, 241-252.

${ }^{50}$ Bagus, P. S.; Sousa, C.; Illas, F. Consequences of Electron Correlation for XPS Binding Energies: Representative Case for C(1s) and O(1s) XPS of CO. J. Chem. Phys. 2016, 145, 144303.

${ }^{51}$ Tantau, L. J.; Islam, M. T.; Payne, A. T.; Chantler, C. T.; Best, S. P.; Tran, C. Q.; Cheah, M. H. High Accuracy Energy Determination and Calibration of Synchrotron Radiation by Powder Diffraction. Radiat. Phys. and Chem. 2014, 95, 73-77. 
Graphic for TOC

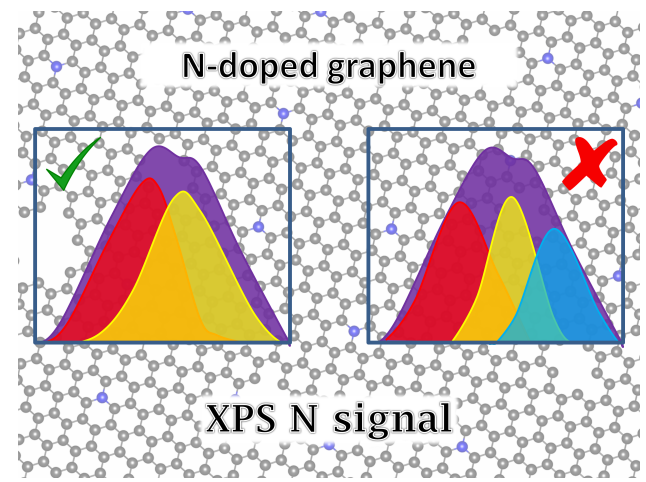

